\title{
Medical Image of the Week: Erythema Nodosum
}

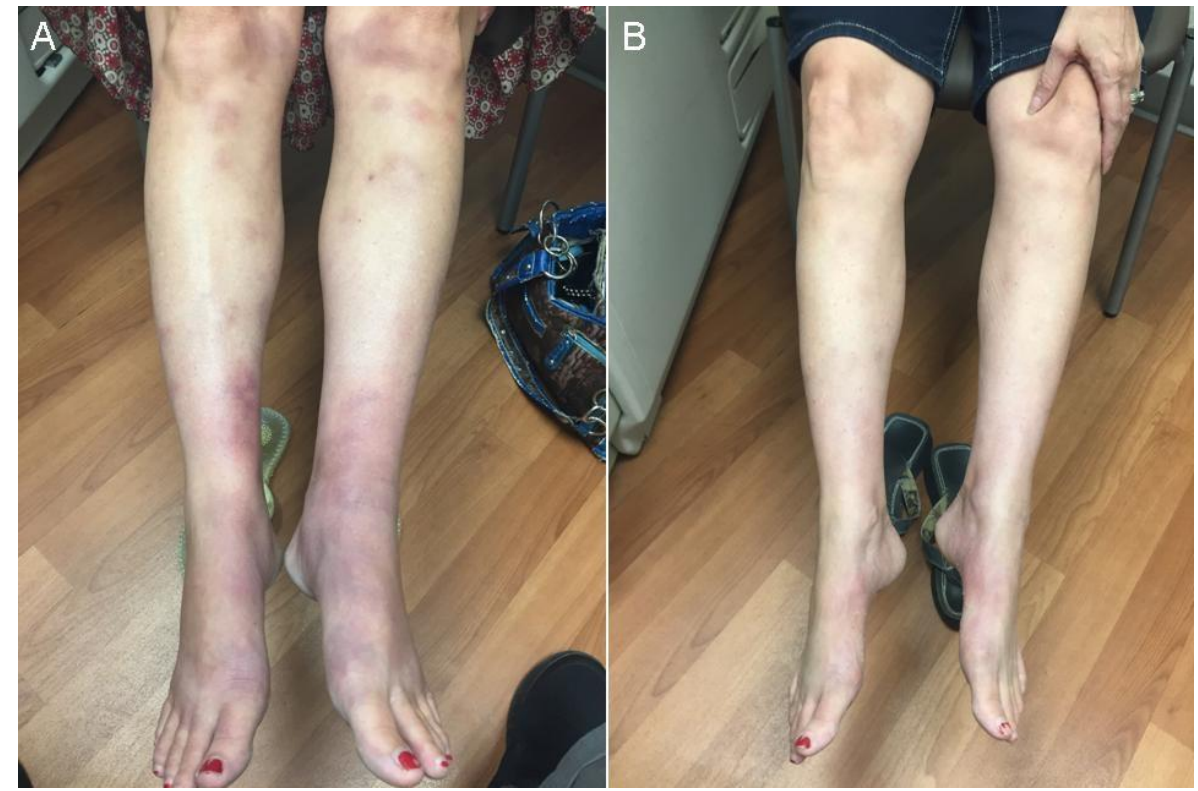

Figure 1. Panel A: photograph of legs at initial presentation. Panel B: 2 weeks later.

A healthy 43-year-old woman presented to the emergency room with one day of diarrhea, vomiting and severe left-sided pleuritic chest pain. Chest radiography revealed an infiltrate in the left lower lobe with a small pleural effusion. White count was 14,000 cells/mcL. Eosinophil count was $2 \%$. She was thought to have pneumonia and treated with azithromycin.

Two weeks later she returned to the emergency room with painful raised erythematous lesions on both lower extremities and generalized joint pain. A thoracic CT scan was performed showing left lower lobe pneumonia and small bilateral pleural effusions. Eosinophilia was $14 \%$. She was diagnosed again as having pneumonia. There was no diagnosis made of the skin lesions. A cocci serology was drawn and sent to Davis California. She was given doxycycline.

Subsequently the cocci serology was found to be positive for IgG and IgM and negative for complement fixation antibodies. She was seen by her primary care physician who diagnosed acute coccidioidomycosis and started her on Diflucan $200 \mathrm{mg}$ daily and referred her for consultation. At her first visit 6 weeks into her illness she was still complaining of arthralgias, fatigue and cough.

Physical examination was negative except for innumerable red raised lesions on her thighs and anterior surfaces of her legs with confluence at the ankles (Figure 1). One month after her initial visit her legs were much improved although there was some peeling of the skin and residual erythema of the lower extremities (Figure 2).

Erythema Nodosum (EN) is a panniculitis of subcutaneous fat which can be associated with a variety of conditions including streptococcal pharyngitis, tuberculosis, sarcoidosis, 
inflammatory bowel disease, cancer, or bacterial infections (1). The usual presentation of this disease is the presence of painful raised erythematous nodules symmetrically on the anterior surfaces of the lower extremities. These lesions do not represent sites of infection but are most likely a result of type IV delayed hypersensitivity.

EN was first described as a benign form of coccidioidomycosis in 1936, and was further characterized by Charles Smith in 1940 when he described acute coccidioidomycosis as an illness characterized by an "influenza like initial phase followed in 2 to 18 days by the eruptive phase of erythema nodosum lasting from six days to three weeks with pigmented areas lasting for months" (2). He noted that recovery was invariable. This was in a time where the frequent presentation of acute coccidioidomycosis as an inapparent infection was not known and the mortality of acute cocci was as high as $50 \%$. Twenty years later, Smith and Pappagiannis made the observation that EN was 2 to 10 times more frequent in females than males (3). Braverman (4) in 1999 observed the protective effect of EN by reporting on 60 pregnant women with coccidioidomycosis. Thirty of these women with EN had no dissemination, and of the 30 without EN, 11 disseminated and one died. The mechanism of this protective effect has yet to be characterized.

Physicians living in the Southwest have learned that "the bumps" (EN), desert rheumatism (polyarthralgia), eosinophilia associated with a flulike illness, and acute knifelike pleuritic chest pain in an otherwise healthy person are all signs and symptoms which lead to a rapid diagnosis of coccidioidomycosis (5). Because of the time sequence of the appearance of EN in this illness this rash is often felt incorrectly to be an allergic reaction to antibiotics given for the previously diagnosed pneumonia. Even though EN is associated with many varied conditions, its presence in the Southwestern United States should lead the physician to consider coccidioidomycosis as the most likely diagnosis.

Gerald F. Schwartzberg, MD

HonorHealth Pulmonology

Phoenix, AZ USA

\section{References}

1. Blake T, Manahan M, Rodins K. Erythema nodosum - a review of an uncommon panniculitis. Dermatol Online J. 2014 Apr 16;20(4):22376. [PubMed]

2. Smith CE. Epidemiology of acute coccidioidomycosis with erythema nodosum ("San Joaquin" or "Valley Fever"). Am J Public Health Nations Health. 1940 Jun;30(6):60011. [CrossRef] [PubMed]

3. Smith CE, Pappagianis D, Levine HB, Saito M. Human coccidioidomycosis. Bacteriol Rev. 1961 Sep;25:310-20. [PubMed]

4. Braverman IM. Protective effects of erythema nodosum in coccidioidomycosis. Lancet. 1999 Jan 16;353(9148):168. [CrossRef] [PubMed]

5. Stevens DA. Coccidioidomycosis. N Engl J Med. 1995 Apr 20;332(16):1077-82. [CrossRef] [PubMed] 


\section{Addendum: Another Erythema Nodosum}

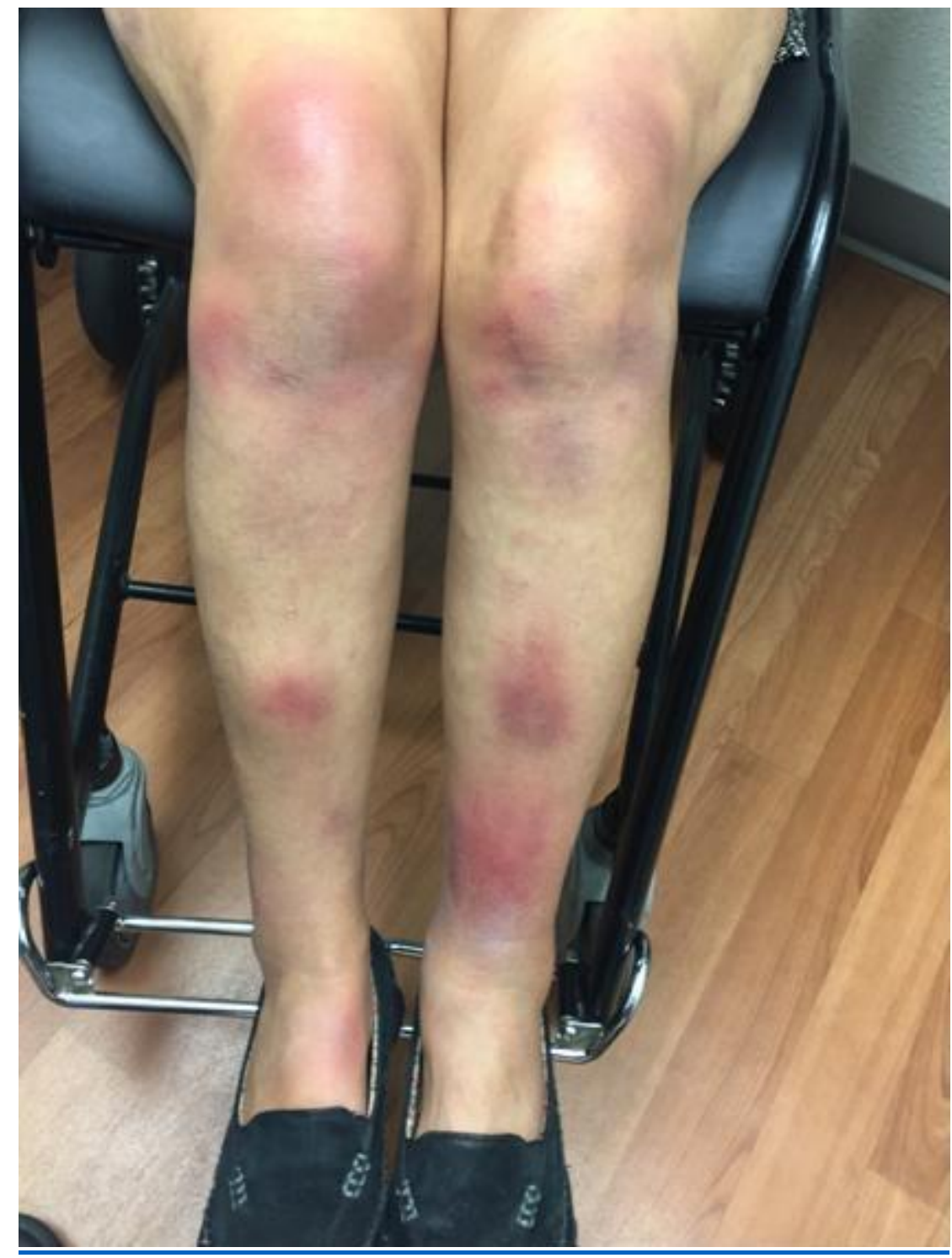

Figure 2. Another case of erythema nodosum.

While the above article was "in press", another case of EN came into the office (Figure 2). Her history was similar to the first patient. Uncle Jun, the patriarch in the "Sopranos," said in his heavy New York accent, "They come in threes." Based on Uncle Jun, I am anticipating seeing another case of EN this week.

Gerald F. Schwartzberg, MD

HonorHealth Pulmonology

Phoenix, AZ USA 